\title{
Delayed adolescent growth in homozygous sickle cell disease
}

\author{
Atul Singhal, Peter Thomas, Robert Cook, Klaas Wierenga, Graham Serjeant
}

\begin{abstract}
Analysis of the growth abnormalities in sickle cell disease has been limited by the lack of longitudinal observations in individuals, and by an inability to quantitate the observed patterns. To investigate the timing and pattern of the adolescent growth spurt, longitudinal observations of height from the Jamaican cohort study were fitted to a mathematical model of growth (Preece-Baines model 1). The study included 44 children with homozygous sickle cell (SS) disease, 44 age and sex matched subjects with sickle cell haemoglobin C (SC) disease, and 44 age and sex matched controls with normal (AA) haemoglobin.

Compared with AA controls, the onset of the adolescent growth spurt was delayed in SS disease by 1.4 years $(95 \%$ confidence interval 0.8 to $2 \cdot 0$ ) with no significant sex difference. The age at peak height velocity was delayed by 1.6 years (0.9 to 2.3) in SS compared with AA subjects but the adolescent growth of SS children was otherwise normal and there was no difference in the attained height by age 17.9 years. The growth spurt was not delayed in SC disease. The age at menarche in girls with SS disease (mean (SD) $15.4(1.3)$ years) was significantly later than girls with SC disease $(13 \cdot 7(1 \cdot 7)$ years) and those with AA haemoglobin (13.1 (1.3) years) but these genotype differences were no longer significant after controlling for the delay in the adolescent growth spurt. The normally coordinated but slightly delayed pattern of growth and normal adult heights suggests a good prognosis for adolescent growth delay in SS disease. Most children with SS disease can therefore be reassured on the outcome of retarded adolescent growth.

(Arch Dis Child 1994; 71: 404-408)
\end{abstract}

Medical Research

Council Laboratories

(Jamaica), University

of the West Indies,

Kingston 7,

Jamaica, WI

A Singhal

P Thomas

R Cook

$\mathrm{K}$ Wierenga

G Serjeant

Correspondence and reprint requests to:

Professor Serjeant.

Accepted 24 July 1994 progress normally. ${ }^{9}$ Cross sectional data, 24 however, fail to account for individual variation in the timing of the adolescent growth

Growth and development is impaired in children with homozygous sickle cell (SS) height. Abnormalities include early de maturation, ${ }^{14-6}$ although a normal adult height suggests a good final height prognosis. ${ }^{7}$ spurt and simply taking means of individual heights at different ages produces a growth curve that is lengthened and reduced in intensity. A more accurate quantitation of the growth defect in SS adolescents has been limited by the lack of longitudinal observations but the height data from the Jamaican cohort study have now allowed assessment of the pattern of adolescent growth.

The study has compared the adolescent growth of children with SS disease with that of children with sickle cell haemoglobin C (SC) disease and with control subjects with a normal (AA) haemoglobin genotype. Longitudinal height data from birth in these individuals have been fitted to a mathematical model (Preece and Baines model 1), ${ }^{10}$ to assess the effect of sickle cell disease on the timing of the adolescent growth spurt. The relationship between timing of the adolescent growth spurt and developmental changes during puberty has also been assessed.

\section{Patients and methods}

PATIENTS

The patients attended the sickle cell clinic of the University Hospital of the West Indies and participated in a cohort study from birth. Patients were ascertained by neonatal screening of 100000 consecutive normal deliveries at the main government maternity hospital (Victoria Jubilee) between July 1973 and December 1981. The diagnostic criteria and techniques have been described. ${ }^{11}$ The first 125 children with SS disease in the cohort study were each matched with two AA children of the same sex, born close in time to the index case. The cohort of 315 children with SS disease, 201 with SC disease, and $250 \mathrm{AA}$ controls (aged 11-18 years at the study date) were followed up prospectively at the sickle cell clinic.

The study was confined to postpubertal children with observations available to the age of 16 years. At the study date, 76/315 SS children should have been eligible from their dates of birth but 21 had died, five had emigrated, and three were excluded because of a chronic disease other than sickle cell disease that might have affected growth. Three males with extreme retardation of sexual maturation, but growing normally for their bone age, were also excluded as they were still prepubertal (Tanner stage 1) and their data could not be analysed using the Preece-Baines model 1. There remained 44 SS patients (mean (SD) age $17.9(0.6)$ years; 21 males, 23 females) 
who were matched by age and sex to the closest 44 cohort children with SC disease $(17.3(0.8)$ years $)$ and to 44 control AA children $(17.9(0.5)$ years $)$.

\section{GROWTH DATA}

Height was measured at three month intervals in SS and SC children and six month intervals in AA controls (aligned with the subject's birthday) using a Harpenden wall mounted stadiometer accurate to $0 \cdot 1 \mathrm{~cm}$ (Holtain Instruments). Computer generated curves for height against age were used to identify outlying values for checking and editing. Sitting height was measured for SS and AA but not SC children at six month intervals using a sitting height table (Holtain Instruments) and subischial length derived from standing height minus sitting height. All measurements were made using standard protocols by clinical nursing and medical staff trained in the techniques involved. ${ }^{12}$

Pubertal development of SS and AA children was assessed by Tanner staging at six month intervals from the age of 8 years using standard photographs as a guide. The first sign of puberty (whether pubic hair or genitalia) was used for analysis as this is likely to be the most accurately recorded. To minimise any bias that may arise from SS children attending more regularly than AA controls, the age at Tanner stage 2 was calculated as the midpoint of the date at which pubertal changes were first observed and the last date at which children were prepubertal. The age at menarche was ascertained by prospective inquiry at each clinic visit. The exact date of menarche was usually available but, if only the month was recalled, the 15th day of that month was used for analysis.

\section{CURVE FITTING}

Height data at six month intervals ( \pm 8 weeks) from the age of 1 year were fitted by computer (using the least squares regression method) to the Preece-Baines model 1 as described below.

$$
h_{(t)}=h_{1}-\frac{2\left(h_{1}-h_{q}\right)}{\exp \left[S_{0}(t-q)\right]+\exp \left[S_{1}(t-q)\right]}
$$

where $h$ is height $(\mathrm{cm})$ at age $t, h_{1}$ is final adult height, $S_{0}$ and $S_{1}$ are rate constants, $q$ is a time constant, and $h_{q}$ is height at $t=q$.

This model has been extensively used to fit longtitudinal data on height ${ }^{13-15}$ and has the advantage over other mathematical models of not requiring the subject's final adult height. The model resolves complex growth curves into a limited number of biologically meaningful parameters defining the adolescent growth curve of each individual and allowing comparison of the timing and degree of the adolescent growth spurt between individuals. The principal indices were the age at onset of the adolescent growth spurt (take off) and the age at peak height velocity. Also derived from the model were four indices of growth (age, height, height velocity, and percentage of adult height) at take off and at peak height velocity, predicted adult height, height gained between take off and peak height velocity, and between peak height velocity and adult height, and change in height velocity between take off to peak height velocity.

\section{STATISTICAL ANALYSIS}

Genotype and sex differences in growth parameters were statistically assessed using the multivariate approach to repeated measures analysis of variance. Type 1 error was fixed at $5 \%$ for each omnibus null hypothesis and the significance level of each pairwise comparison of genotypes was adjusted using Bonferroni's method. The mean values of the mathematical parameters describing individual curves were used to produce a mean constant curve for each genotype and sex group. For each individual, all the differences between observed and fitted points (residuals) were used to calculate a standard deviation of residuals. A small standard deviation indicates a better fit to the model.

\section{Results}

CURVE FITTING

The model fitted the growth data of AA, SS, and SC subjects equally well and the mean of the standard deviation of the residuals did not differ significantly between genotypes (table 1).

\section{PATTERN OF ADOLESCENT GROWTH}

The indices of adolescent height growth in the three genotypes are summarised in table 2 . Compared with AA controls, SS patients showed a delay in mean age at take off of 1.4 years ( $95 \%$ confidence interval 0.8 to 2.0 ), a delay in mean age at peak height velocity of 1.6 years $(0.9$ to 2.3$)$, and a lower height velocity at take off. Comparable differences in SC patients compared with AA controls did not reach significance. The mean predicted height and height velocity against age for males and females using fitted data (mean constant curve) are shown in the figure. There were significant sex differences in growth indices in all genotypes.

The sitting height to subischial length ratio (available in 29 and 27 SS/AA pairs at age 16 and 8 years respectively) was significantly lower in SS subjects (mean (SD) $0.95(0.053)$ ) than AA controls $(0.99(0.065) ; \mathrm{p}=0.010)$ at age 16 years, but not at 8 years of age $(p=0 \cdot 29)$. There was no sex differences in this ratio.

Table 1 Residuals data for fitting Preece-Baines model 1 to height; figures are mean (SD)

\begin{tabular}{clccl}
\hline & $A A$ & $S S$ & $S C$ \\
\hline No of data & $\mathrm{M}$ & $24 \cdot 5(4 \cdot 2)$ & $25 \cdot 8(5 \cdot 3)$ & $21 \cdot 3(5 \cdot 6)$ \\
points & $\mathrm{F}$ & $24 \cdot 1(3 \cdot 8)$ & $25.3(5 \cdot 8)$ & $22 \cdot 3(6 \cdot 3)$ \\
SD of resi- & $\mathrm{M}$ & $0 \cdot 75(0 \cdot 18)$ & $0 \cdot 79(0 \cdot 19)$ & $0 \cdot 80(0 \cdot 29)$ \\
duals (cm) & $\mathrm{F}$ & $0 \cdot 87(0 \cdot 26)$ & $0.94(0.32)$ & $0.70(0 \cdot 25)$ \\
\hline
\end{tabular}

SC patients had significantly fewer $(p<0.05)$ data points than SA patients had signific
AA and subjects. 
Table 2 Adolescent height growth in sickle cell disease (repeated measures analysis of variance); figures are mean (SD)

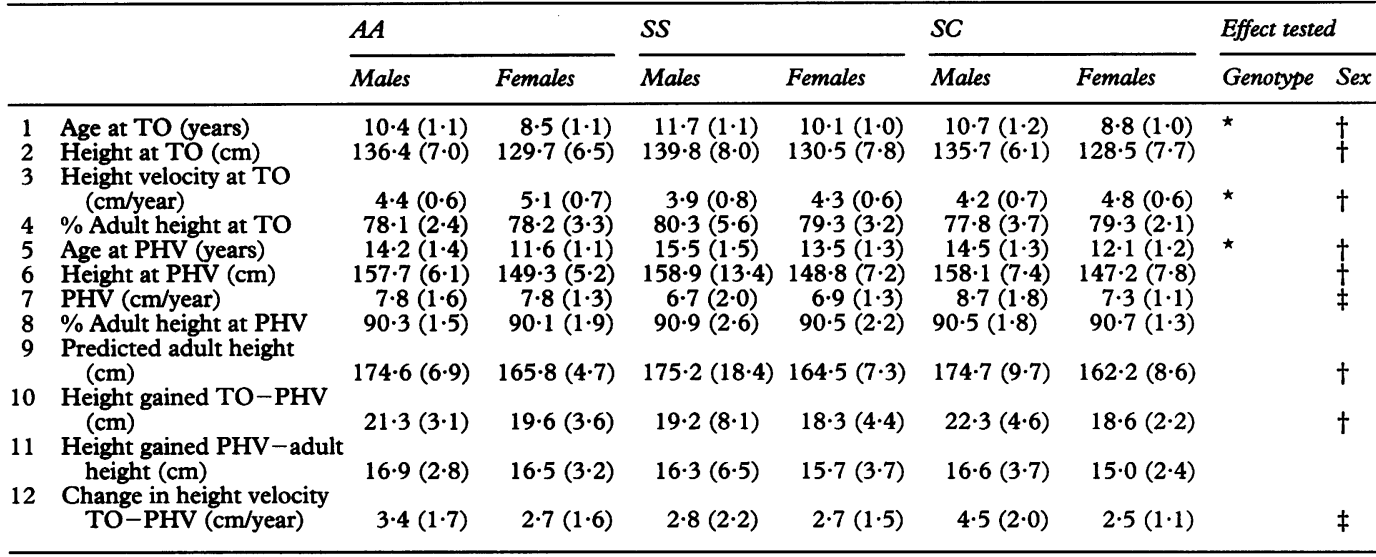

$\mathrm{PHV}=$ peak height velocity; $\mathrm{TO}=$ take off.

$\star$ Overall genotype difference, $\mathrm{p}<0.001$. Paired comparisons showed differences were between AA $v$ SS and SC $v$ SS tp $<0.05$; ‡within SC genotype only, $p<0.05$.

At the last clinic visit $41 \mathrm{SS}, 42 \mathrm{SC}$, and 43 AA subjects had a height difference of $<1 \mathrm{~cm}$ /year over the preceding year. Three SS and two SC patients were still growing faster than $1 \mathrm{~cm} /$ year and one AA subject had died, leaving 39 matched SS, SC, and AA subjects with similar minimal rates of growth. In this group there was no significant genotype difference in height (repeated measures analysis of variance: $p=0 \cdot 89$ ).

PATTERN OF ADOLESCENT DEVELOPMENT The first pubertal changes (Tanner stage 2) appeared later in SS subjects (mean (SD) males: $12.8(1.6)$ years; females: $12.0(1.8)$ ) than in AA controls (males: $11.1(1 \cdot 2)$ females: $10 \cdot 1(1 \cdot 2)$ ) for the 42 pairs with available data (repeated measures analysis of variance, $\mathrm{p}<0.001$ ) but adjusting for the age at take off or peak height velocity reduced the mean genotype difference from 1.8 years to 1.2 years (95\% confidence interval 0.5 to 2.0$)$ and to 1.2 years $(0.4$ to 1.9$)$ respectively. The delay in onset of puberty in SS patients compared with their AA controls correlated with the delay in age at peak height velocity $(r=0.36$, $\mathrm{p}=0.02$ ) but the correlation with age at take off just failed to reach significance $(r=0.30$, $\mathrm{p}=0.054)$. In both genotypes, boys started puberty later $(p=0.01)$ than girls. There were no Tanner stage data for children with SC disease.

The age at menarche in girls with SS disease (mean (SD) 15.4 (1.3) years) was significantly later than girls with SC disease (13.7 (1.7) years) and those with a normal AA haemoglobin genotype $(13.1(1.3)$ years; $p<0.001$ for the
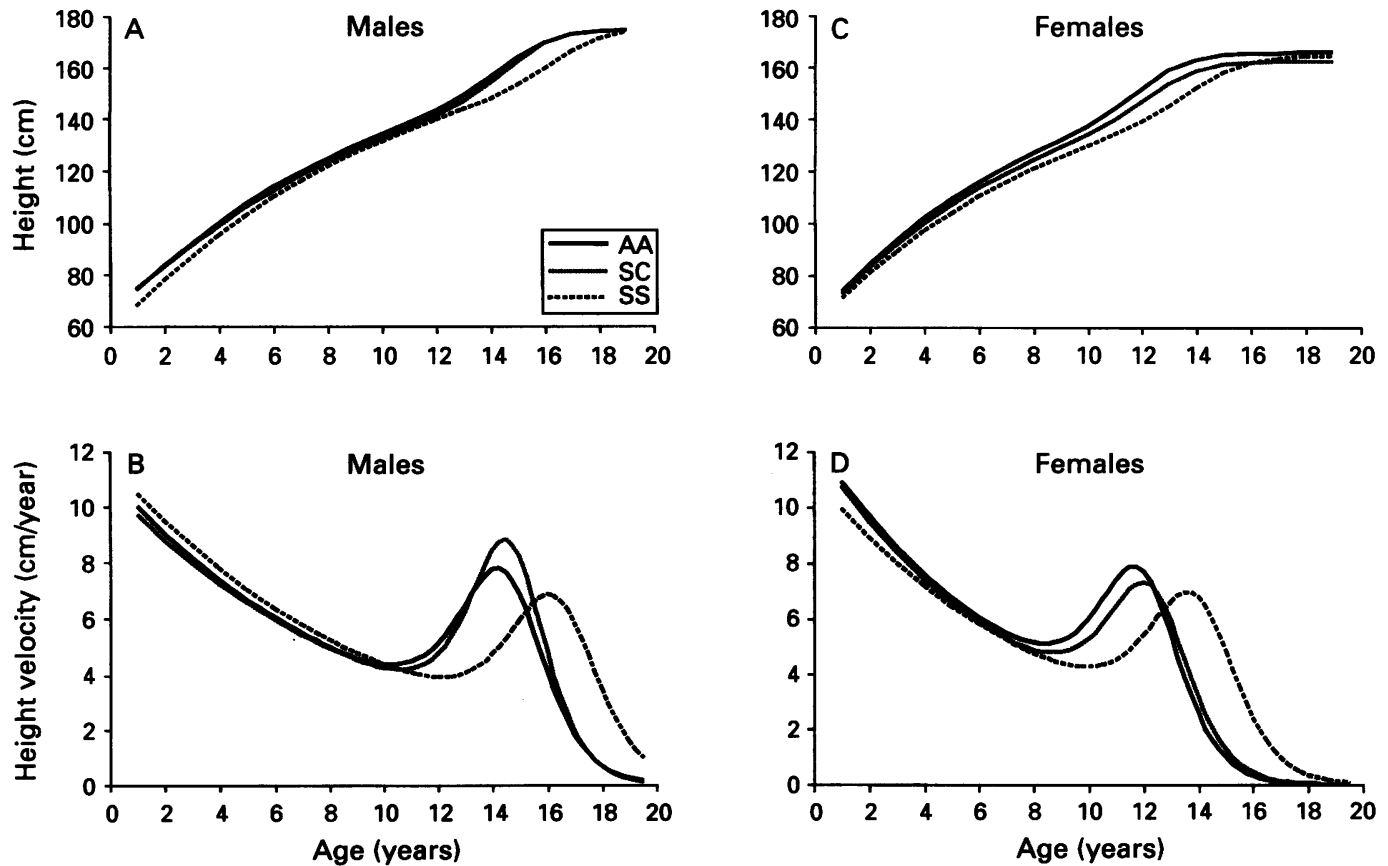

The mean height and height velocity for males ( $A$ and $B$ ) and females ( $C$ and $D$ ) derived from the means of individual data fitted to the Preece-Baines model 1. 
overall genotype comparison). However, after adjusting for the delay in the adolescent growth spurt (by using age at take off or peak height velocity as a covariate in repeated measures analysis of variance) the genotype difference in age at menarche was no longer significant (the $2 \cdot 3$ year difference between SS and AA girls was reduced to 1.0 years $(95 \%$ confidence interval $-0 \cdot 1$ to $2 \cdot 0$ ) after adjusting for age at take off and to 0.6 years $(-0.4$ to 1.6$)$ after adjusting for age at peak height velocity)). The genotype differences in age at menarche between $S S$ and $\mathrm{AA}, \mathrm{SS}$ and SC, and AA and SC pairs were highly correlated with the genotype differences in age at take off or peak height velocity (correlations ranged from 0.72 to $0.87, \mathrm{p}<0.001$ for all six comparisons).

\section{Discussion}

Interpretation of growth data from any population depends on the sample selected for investigation and the study design. Subjects from the cohort study were diagnosed at birth and should be a representative sample of children with sickle cell disease. Analysis of longitudinally collected height data using the Preece-Baines model 1 allowed assessment of individual variation in timing of the adolescent growth spurt and hence comparison with adolescent growth in the three genotypes. The quality of curve fitting in the present study was not as good as in previous applications of the model, the pooled residual mean squares of $0.9 \mathrm{~cm}^{2}$ contrasting with figures of $0.19 \mathrm{~cm}^{2}$ in the study by Preece and Baines, ${ }^{10}$ and $0 \cdot 46,{ }^{13}$ $0.40,{ }^{14}$ and $0.35^{15}$ in subsequent studies. A factor contributing to this variability may have been the analysis of data from as early as 1 year of age (as growth delay in SS disease is apparent as early as age 6 months), although better fits to the model may be obtained by restricting to data at later ages. ${ }^{14}$ Other factors may include the greater intrinsic inaccuracy in measuring young children, measurement error due to observer variation over the study span of 18 years, and technical variation arising from changing hairstyles.

The present data confirm delay in the adolescent growth spurt of SS children. The true delay in males may have been underestimated as three SS boys were excluded as their growth spurt had not started by the age of 16 years and therefore their pattern of adolescent growth could not be assessed. Adolescent growth in SS disease was otherwise normal and as height velocity falls before the adolescent growth spurt, the lower height velocity at take off probably reflects the delay in take off. Furthermore, the lack of significant differences in attained height by age 17 years of SS, SC, and AA subjects is in agreement with cross sectional data on the heights of Jamaican SS adults, ${ }^{7}$ and consistent with delayed epiphyseal fusion, ${ }^{5}$ allowing a catch up in height. The more normal growth of SC compared with SS children has been reported, 12 and is confirmed by the height velocity growth curves. The later adolescent growth spurt of boys compared with girls of all genotypes was consistent with their later physical and sexual maturation ${ }^{16}$ but the pattern of adolescent growth and the degree of growth delay was similar in both sexes with SS disease.

The delay in onset of menarche in SS disease was appropriate for the overall retardation in adolescent growth, but the delay in appearance of the first pubertal changes persisted even after controlling for the later adolescent growth spurt. The reduced spinal (sitting) to subischial length in SS children at the age of 16 years was characteristic of SS and normal children with delayed adolescent growth. ${ }^{17}$

The aetiology of retarded growth in SS disease is probably multifactorial with contributions from abnormal endocrine function, ${ }^{6}$ hypogonadism (particularly in males), ${ }^{18}$ suboptimal nutrition, ${ }^{19}$ and an increase in metabolism, ${ }^{20}$ possibly as a result of the high rate of erythropoiesis in this condition. A normal but delayed pattern of adolescent growth in most SS children suggests that these factors affect the timing of the adolescent growth spurt but not the final height. Children with extreme growth delay may represent an interesting subgroup who should be investigated for a possible endocrine explanation for their delayed puberty. However, most SS children with retardation of adolescent growth and development can be reassured as to their final height prognosis.

We thank Dr Peter S W Davies for technical assistance and advice.

1 Stevens MCG, Maude GH, Cupidore L, Jackson H, Hayes RJ, Serjeant GR. Prepubertal growth and skeletal maturation in children with sickle cell disease. Pediatrics 1986; 78: 124-32.

2 Platt OS, Rosenstock W, Espeland MA. Influence of sickle hemoglobinopathies on growth and development. $N$ Engl 7 Med 1984; 311: 7-12

3 Phebus CK, Gloninger MF, Maciak BJ. Growth patterns by age and sex in children with sickle cell disease. $\mathcal{f}$ Pediatr 1984; 105: 28-33.

4 Ashcroft MT, Serjeant GR, Desai P. Heights, weights and skeletal age of Jamaican adolescents with sickle cell anaemia. Arch Dis Child 1972; 47: 519-24.

5 Serjeant GR, Ashcroft MT. Delayed skeletal maturation in sickle cell anemia in Jamaica. Fohns Hopkins Medical fournal 1973; 132: 95-102.

6 Olambiwonnu NO, Penny R, Frasier SD. Sexual maturation in subjects with sickle cell anemia: studies of serum gonadotropin concentration, height, weight and skeletal gonadotropin concentration, heigh

7 Ashcroft MT, Serjeant GR. Body habitus of Jamaican adults with sickle cell anemia. South Med f 1972; 65: 579-82.

8 Graham C, Maude GH, Serjeant GR. Delayed menarche in homozygous sickle cell disease. West Indian Med $\mathcal{F} 1986$; 35: $18-22$.

9 Luban NLC, Leikin SL, August GA. Growth and development in sickle cell anemia. Am $\mathcal{f}$ Pediatr Hematol Oncol 1982; 4: 61-5.

10 Preece MA, Baines MJ. A new family of mathematical models describing the human growth curve. Ann Hum Biol 1978; 5: 1-24.

11 Serieant BE, Forbes $M$, Williams LL Serieant GR Screening cord bloods for detection of sickle cell disease in Jamaica. Clin Chem 1974; 20: 666-9.

12 Cameron N. The Measurement of Human Growth. Croom Helm, 1984

13 Hauspie RC, Das SR, Preece MA, Tanner JM. A longitudinal study of the growth in height of boys and girls of West Bengal (India) aged six months to 20 years. Ann Hum Biol 1980; 7: 429-41.

14 Hauspie RC, Wachholder A, Baron G, Cantraine F, Susanne C, Graffar M. A comparative study of the fit of four different functions to longitudinal data of orowth in height of Belgian girls. Ann Hum Biol 1980; 7; 347-58.

15 Mirwald RL, Bailey DA, Cameron N, Rasmussen RL. Longitudinal comparison of aerobic power in active and inactive boys aged 7.0 to 17.0 years. Ann Hum Biol 1981; 8: $405-14$.

16 Tanner JM. Sex differences in physique. Growth at adolescence. 2nd Ed. London: Blackwell Scientific, 1962: adolescence. 
17 Stanhope R, Albanese A, Shalet S. Delayed puberty. Many good arguments to treat. $B M F$ 1992; 305: 790.

18 Odonkor PO, Addae SK. Circulating testosterone in adolescent and young adult sickle cell disease patients. IRCS 1982;10: 302 .

19 Heyman MB, Vichinsky E, Katz R, et al. Growth retarda- tion in sickle cell disease treated by nutritional support. Lancet 1985; ii: 903-6.

20 Singhal A, Davies P, Sahoota A, Thomas PW, Serjeant GR. Resting metabolic rate in homozygous sickle cell disease. Am f Clin Nutr 1993; 57: 32-4.

\section{Why do they wheeze so much?}

Atmospheric pollutants which may affect respiratory health include smoke, sulphur dioxide $\left(\mathrm{SO}_{2}\right)$, ozone, oxides of nitrogen, sulphate, hydrogen ion, and carbon monoxide. A paper from Birmingham (Sarah Walters and colleagues, Thorax $1994 ; 49: 133-40$ ) reports on the relationship between air concentrations of smoke and $\mathrm{SO}_{2}$ and hospital admissions of people of all ages for asthma and other respiratory diseases in the city during the two years ending March 1990.

Despite the fact that the concentrations of smoke and $\mathrm{SO}_{2}$ were within EEC guidelines throughout (apart from one day) there was a significant association between these concentrations and daily hospital admissions in winter for all respiratory illnesses and for asthma. Mean (maximum) daily air concentrations of smoke were $12 \cdot 7(188 \cdot 3) \mu \mathrm{g} / \mathrm{m}^{3}$ and of $\mathrm{SO}_{2} 39 \cdot 1(126 \cdot 3)$ $\mu \mathrm{g} / \mathrm{m}^{3}$. It was estimated that in winter a rise in smoke concentration of $100 \mu \mathrm{g} / \mathrm{m}^{3}$ might result in 21 more admissions to Birmingham hospitals each day for respiratory illnesses including five for asthma. An identical rise in $\mathrm{SO}_{2}$ might give four more asthma and 15 more total respiratory admissions. The increase in admissions tended to follow the increase in pollution by about two days. No separate information is given about children but in an American study increased air pollution resulted in a $47 \%$ rise in hospital admissions for adults and an $87 \%$ rise for children. ${ }^{1}$ It seems therefore that short term increases in smoke and $\mathrm{SO}_{2}$ air pollution are associated with increased respiratory morbidity even when the levels remain within EEC suggested limits. Nevertheless, there has been no increase in such pollution over the last 30 years and it cannot explain the increased prevalence of asthma in recent years.

So why is asthma on the increase? The question is considered by Anthony Seaton and co-writers in the same issue of Thorax $(1994 ; 49: 171-4)$. In the last 20 years the prevalence of both asthma and eczema has doubled in Britain and hay fever has become three or four times more common. In Australia and New Zealand there has also been a twofold increase in childhood asthma. Yet atmospheric pollution in Britain does not compare with that of the 1950s. There has been no increase in any measured pollutant adequate to explain the epidemiological data. Neither has there been a rise in the main indoor air pollutant, tobacco smoke, or in exposure to house dust mite or domestic animals.

There is evidence that where societies have become 'westernised' and where people have migrated to westernised societies an increase in the prevalence of asthma has followed. Over the relevant period there has been a change in British dietary habits and these authors argue that this change could be the cause of the increase in atopic disease. In the 25 years up to 1985 people in Britain ate considerably less fresh fruit and vegetables, potatoes, meat, and fish. These foods are all important sources of antioxidants. The suggestion is that the increase in disease could be a result of the effects of oxygen free radicals unopposed by natural antioxidants. It is clearly a hypothesis which needs to be addressed. We are in the middle of a major epidemic which we can no longer tolerate with any degree of equanimity. Who is going to remove the handle from the parish pump of this disease?

ARCHIVIST 